\title{
Conservation Laws and Hamilton's Equations for Systems with Long-Range Interaction and Memory
}

\author{
Vasily E. Tarasov ${ }^{1,2}$, George M. Zaslavsky ${ }^{1,3}$ \\ 1) Courant Institute of Mathematical Sciences, New York University \\ 251 Mercer St., New York, NY 10012, USA \\ 2) Skobeltsyn Institute of Nuclear Physics, \\ Moscow State University, Moscow 119992, Russia \\ 3) Department of Physics, New York University, \\ 2-4 Washington Place, New York, NY 10003, USA
}

\begin{abstract}
Using the fact that extremum of variation of generalized action can lead to the fractional dynamics in the case of systems with long-range interaction and long-term memory function, we consider two different applications of the action principle: generalized Noether's theorem and Hamiltonian type equations. In the first case, we derive conservation laws in the form of continuity equations that consist of fractional time-space derivatives. Among applications of these results, we consider a chain of coupled oscillators with a power-wise memory function and power-wise interaction between oscillators. In the second case, we consider an example of fractional differential action 1-form and find the corresponding Hamiltonian type equations from the closed condition of the form.
\end{abstract}




\section{Introduction}

Different physical phenomena such as anomalous transport or random walk with infinite moments [1, 2], dynamics of porous media [3, 4], continuous time random walk [5, 6, 7], chaotic dynamics [8] (see also reviews [9, 10]) can be described by equations with fractional integrodifferentiation. Despite of fairly deep and comprehensive results in fractional calculus (see [11, 12, 13, 14]) a possibility of their applications to physics needs to develope specific physical tools such as extension of fractional calculus to the areas as multi-dimension [11, 17], multi-scaling [15, 16], variational principles [18, 19]. It became recently formalized the links of the dynamical equations with fractional derivatives to the systems with long-range interaction [20, 21, 22] and long-term memory [23].

In this paper, we concentrate on two problems important for numerous physical applications: conservation laws and Hamiltonian type equations, both obtained from the corresponding fractional action principles. In Sec.2, we derive the Noether's theorem for a Lagrangian that includes non-local space-time densities. The Noether's theorem was also discussed in [26, 27]. Our new derivation shows in an explicit way how fractional derivative in time emerges from the specific type of the memory function, and how fractional derivative in space is related to a specific long-distance potential of interaction (Sec. 3.) In Sec. 4, these results are applied to a chain of nonlinear oscillators that is a subject of great interest in statistics and dynamics [28, 29]. Finally, at Sec.5, we derive a specific case of fractional Hamilton's equations. Different steps in this direction were performed in [32, 33, 34. We consider the Lagrangian density as a functional without fractional derivatives but, instead, the differential 1-form has fractional differentials. Some examples are given for this type of systems.

The main feature of this paper is the consideration of fractional type differentials or derivatives in both space-time coordinates. 


\section{Noether's theorem for long-range interaction and mem- ory}

\subsection{Action and Lagrangian functionals}

Let us consider the action functional

$$
S[u]=\int_{R} d^{2} x \int_{R} d^{2} y \mathcal{L}(u(x), u(y), \partial u(x), \partial u(y)),
$$

where $x=(t, r), t$ is time, $r$ is coordinate, and $y=\left(t^{\prime}, r^{\prime}\right), \partial u(x)=\left(\partial_{t} u(t, r), \partial_{r} u(t, r)\right)$. The integration is carried out over a region $R$ of the 2 -dimensional space $\mathbb{R}^{2}$ to which $x$ belong. The field $u(x)$ is defined in the region $R$ of $\mathbb{R}^{2}$. We assume that $u(x)$ has partial derivatives

$$
\partial_{0} u(x)=\frac{\partial u(t, r)}{\partial t}, \quad \partial_{1} u(x)=\frac{\partial u(t, r)}{\partial r},
$$

which are smooth functions with respect to time and coordinate. Here $\mathcal{L}(u(x), u(y), \partial u(x), \partial u(y))$ is generalized density of Lagrangian. If

$$
\mathcal{L}(u(x), u(y), \partial u(x), \partial u(y))=\mathcal{L}(u(x), \partial u(x)) \delta(x-y),
$$

then we have the usual action functional

$$
S[u]=\int_{R} d^{2} x \mathcal{L}(u(x), \partial u(x)) .
$$

The variation of the action (1) is

$$
\begin{aligned}
\delta S[u, h]= & \int_{R} d^{2} x \int_{R} d^{2} y\left(\frac{\partial \mathcal{L}}{\partial u(x)} h(x)+\frac{\partial \mathcal{L}}{\partial\left(\partial_{\mu} u(x)\right)} \partial_{\mu} h(x)+\right. \\
& \left.+\frac{\partial \mathcal{L}}{\partial u(y)} h(y)+\frac{\partial \mathcal{L}}{\partial\left(\partial_{\mu} u(y)\right)} \partial_{\mu} h(y)\right),
\end{aligned}
$$

where $\mu=0,1, \partial_{\mu}=\partial / \partial x^{\mu}$ and

$$
\mathcal{L}=\mathcal{L}(u(x), u(y), \partial u(x), \partial u(y))
$$

and $h(x)=\delta u(x)$ is the variation of the field $u$. The variation (3) can be presented as

$$
\delta S[u, h]=\int_{R} d^{2} x \int_{R} d^{2} y\left(\frac{\partial \mathcal{L}_{s}}{\partial u(x)} h(x)+\frac{\partial \mathcal{L}_{s}}{\partial\left(\partial_{\mu} u(x)\right)} \partial_{\mu} h(x)\right),
$$


where

$$
\mathcal{L}_{s}=\mathcal{L}(u(x), u(y), \partial u(x), \partial u(y))+\mathcal{L}(u(y), u(x), \partial u(y), \partial u(x))
$$

We can define the functional

$$
L[x, u, \partial u]=\frac{1}{2} \int_{R} d^{2} y \mathcal{L}_{s}
$$

which will be called the Lagrangian functional. For (2), the functional (5) is equal to the usual Lagrangian density $\mathcal{L}(u, \partial u)$.

Using notations

$$
\frac{\delta L[x, u, \partial u]}{\delta u(x)}=\int_{R} d^{2} y \frac{\partial \mathcal{L}_{s}}{\partial u(x)}, \quad \frac{\delta L[x, u, \partial u]}{\delta\left(\partial_{\mu} u(x)\right)}=\int_{R} d^{2} y \frac{\partial \mathcal{L}_{s}}{\partial\left(\partial_{\mu} u(x)\right)},
$$

the variation (41) is

$$
\delta S[u, h]=\int_{R} d^{2} x\left(\frac{\delta L[x, u, \partial u]}{\delta u(x)} h(x)+\frac{\delta L[x, u, \partial u]}{\delta\left(\partial_{\mu} u(x)\right)} \partial_{\mu} h(x)\right) .
$$

The structure of (77) is non-local, i.e., it includes, through (15) and $\mathcal{L}_{s}$ a possibility of the longterm memory and long-range interaction. Let us derive the generalization of the Noether's theorem for this case in the way analogical to the local case (2).

\subsection{Equations of motion}

First, we separate the variation that is linked in the variation of coordinates

$$
x^{\mu} \rightarrow x^{\prime \mu}=x^{\mu}+\delta x^{\mu},
$$

and the variation caused by a change of the form of $u$,

$$
u(x) \rightarrow u^{\prime}(x)=u(x)+\delta u(x)
$$

The variation $h(x)=\delta u(x)$ of $u(x)$ that is not due to the variation in coordinates is called local. The total variation is

$$
\Delta u(x)=u^{\prime}\left(x^{\prime}\right)-u(x)=\delta u(x)+\left(\partial u / \partial x^{\mu}\right) \delta x^{\mu}
$$


in the first approximation with respect to $\delta x$. Let us consider the variation of (11) as

$$
\delta S[u, h]=\int_{R} d^{2} x^{\prime} L\left[x^{\prime}, u^{\prime}, \partial^{\prime} u^{\prime}\right]-\int_{R} d^{2} x L[x, u, \partial u] .
$$

The elements of the two-dimensional volume in new and old coordinates are related through the formula

$$
d^{2} x^{\prime}=J\left(x^{\prime} / x\right) d^{2} x
$$

where $J\left(x^{\prime} / x\right)=\operatorname{det}\left|\partial x^{\prime \mu} / \partial x^{\nu}\right|$ is the Jacobian of the transformation. Using the well-known relation

$$
\operatorname{det} A=\exp \operatorname{Tr} \ln A
$$

and the linear approximation

$$
\frac{\partial x^{\mu \prime}}{\partial x^{\nu}}=\delta_{\nu}^{\mu}+\partial_{\nu}\left(\delta x^{\mu}\right)
$$

we get

$$
J\left(x^{\prime} / x\right)=1+\partial_{\mu}\left(\delta x^{\mu}\right)
$$

Here $\delta_{\nu}^{\mu}$ is the Kronecker symbol.

For the variation (10), we get

$$
\begin{gathered}
\delta S[u, h]=\int_{R} d^{2} x\left(\delta L+L \partial_{\mu}\left(\delta x^{\mu}\right)\right)= \\
=\int_{R} d^{2} x\left(\frac{\delta L}{\delta u} \delta u+\frac{\delta L}{\delta\left(\partial_{\mu} u\right)} \delta\left(\partial_{\mu} u\right)+\partial_{\mu}\left(L \delta x^{\mu}\right)\right),
\end{gathered}
$$

where the variations of $L$ are defined in (66). Using $\delta\left(\partial_{\mu} u\right)=\partial_{\mu}(\delta u)$, and

$$
\frac{\delta L}{\delta\left(\partial_{\mu} u\right)} \partial_{\mu} \delta u=\partial_{\mu}\left(\frac{\delta L}{\delta\left(\partial_{\mu} u\right)} \delta u\right)-\partial_{\mu}\left(\frac{\delta L}{\delta\left(\partial_{\mu} u\right)}\right) \delta u
$$

we can rewrite (12) as

$$
\begin{gathered}
\delta S[u, h]=\int_{R} d^{2} x\left(\frac{\delta L}{\delta u}-\partial_{\mu} \frac{\delta L}{\delta\left(\partial_{\mu} u\right)}\right) \delta u+ \\
+\int_{R} d^{2} x \partial_{\mu}\left(L \delta x^{\mu}+\frac{\delta L}{\delta\left(\partial_{\mu} u\right)} \delta u\right) .
\end{gathered}
$$


The Gauss theorem gives

$$
\int_{R} d^{2} x \partial_{\mu}\left(L \delta x^{\mu}+\frac{\delta L}{\delta\left(\partial_{\mu} u\right)} \delta u\right)=\int_{\partial R} d S_{\mu}\left(L \delta x^{\mu}+\frac{\delta L}{\delta\left(\partial_{\mu} u\right)} \delta u\right) .
$$

We assume that at the boundary of the domain of integration the function $u(x)$ is selected in a definite manner (the boundary condition). Then stationary (a minimum or a saddle point) values of $S[u]$ from variational equations $\delta S[u, h]=0$ with

$$
[\delta u]_{\partial R}=0, \quad[\delta x]_{\partial R}=0
$$

at the boundary $\partial R$ of the domain of integration, constitutes the necessary and sufficient condition for the real evolution of the field, that is, that $u=u(x)$ represents the true dynamics under the given boundary conditions. The stationary action principle gives

$$
\frac{\delta L[x, u, \partial u]}{\delta u(x)}-\partial_{\mu} \frac{\delta L[x, u, \partial u]}{\delta\left(\partial_{\mu} u(x)\right)}=0
$$

where the variations of $L$ are defined in (6). This equation is the Euler-Lagrange equation for Lagrangian functional $L[x, u, \partial u]$.

Let us consider three special cases of equation (15).

(1) The absence of the memory and long-range interaction means that

$$
\mathcal{L}(u(x), u(y), \partial u(x), \partial u(y))=\mathcal{L}(u(x), \partial u(x)) \delta(x-y)
$$

Then (15) gives the usual Euler-Lagrange equation

$$
\frac{\partial \mathcal{L}(u, \partial u)}{\partial u(x)}-\partial_{\mu} \frac{\partial \mathcal{L}(u, \partial u)}{\partial\left(\partial_{\mu} u(x)\right)}=0 .
$$

(2) If the generalized Lagrangian density is

$$
\mathcal{L}(u(x), u(y), \partial u(x), \partial u(y))=\mathcal{L}(u(x), \partial u(x)) c_{1}(D, r) \delta(x-y)
$$

where

$$
c_{1}(D, r)=\frac{|r|^{D-1}}{\Gamma(D)}, \quad(0<D<1)
$$

for a medium distributed on $\mathbb{R}^{1}$ with the fractional Hausdorff dimension $D$, then Eq. (15) has the form

$$
c_{1}(D, r) \frac{\partial \mathcal{L}(u, \partial u)}{\partial u(x)}-\partial_{\mu}\left(c_{1}(D, r) \frac{\partial \mathcal{L}(u, \partial u)}{\partial\left(\partial_{\mu} u(x)\right)}\right)=0
$$


This is Euler-Lagrange equation for the field $u(x)=u(t, r)$ in fractal medium. Examples of the field (wave) equations for fractal medium string and fractional hydrodynamics are considered in [36]. For example,

$$
\mathcal{L}(u(x), \partial u(x))=\frac{1}{2}\left(\partial_{t} u(t, r)\right)^{2}-\frac{1}{2} v^{2}\left(\partial_{r} u(t, r)\right)^{2},
$$

leads to the equation

$$
c_{1}(D, r) \partial_{t}^{2} u(t, r)-v^{2} \partial_{r}\left(c_{1}(D, r) \partial_{r} u(t, r)\right)=0
$$

that describes the propagation waves in fractal medium.

(3) Consider the action functional

$$
S[u]=\int_{R} d^{2} x \int_{R} d^{2} y\left(\frac{1}{2} \partial_{t} u(x) g_{0}(x, y) \partial_{t^{\prime}} u(y)-\frac{1}{2} \partial_{r} u(x) g_{1}(x, y) \partial_{r^{\prime}} u(y)-V(u(x), u(y))\right),
$$

where the kernels $g_{0}(x, y)$ and $g_{1}(x, y)$ are responsible for nonlocal time-coordinates dynamics and $V$ is nonlocal interaction potential. Then the Lagrangian functional (5) is

$$
\begin{gathered}
L\left[x, u, \partial_{t} u, \partial_{r} u\right]= \\
=\int_{R} d^{2} y\left(\frac{1}{2} \partial_{t} u(x) K_{0}(x, y) \partial_{t^{\prime}} u(y)-\frac{1}{2} \partial_{r} u(x) K_{1}(x, y) \partial_{r^{\prime}} u(y)-U(u(x), u(y))\right),
\end{gathered}
$$

where

$$
\begin{gathered}
K_{0}(x, y)=\frac{1}{2}\left[g_{0}(x, y)+g_{0}(y, x)\right], \\
K_{1}(x, y)=\frac{1}{2}\left[g_{1}(x, y)+g_{1}(y, x)\right], \\
U(u(x), u(y))=V(u(x), u(y))+V(u(y), u(x)) .
\end{gathered}
$$

Assume that

$$
U(u(x), u(y))=U(u(x)) \delta(x-y)
$$

In this case, the Euler-Lagrange functional equation (15) has the form (see also [23])

$$
\int_{R} d^{2} y \partial_{t} K_{0}(x, y) \partial_{t^{\prime}} u(y)-\int_{R} d^{2} y \partial_{r} K_{1}(x, y) \partial_{r^{\prime}} u(y)+\frac{\partial U(u(x))}{\partial u(x)}=0 .
$$


It is an integro-differential equation, which allows us to derive field equations for different cases of the kernels $K_{0}(x, y)$ and $K_{1}(x, y)$.

In the absence of memory and for local interaction the kernels (20) are defined at the only instant $t$ and point $r$, i.e.,

$$
K_{0}(x, y)=g_{0} \delta(x-y), \quad K_{1}(x, y)=g_{1} \delta(x-y)
$$

with some constants $g_{0}$ and $g_{1}$. Then equation (21) gives

$$
g_{0} \partial_{t}^{2} u(t, r)-g_{1} \partial_{r}^{2} u(t, r)+\frac{\partial U(u(t, r))}{\partial u(t, r)}=0
$$

For example $g_{0}=g_{1}=1$, when

$$
U(u(t, r))=-\cos u(t, r)
$$

we get the sine-Gordon equation

$$
\partial_{t}^{2} u(t, r)-\partial_{r}^{2} u(t, r)+\sin u(t, r)=0
$$

\subsection{Noether's current}

Let us derive the Noether's current by using the action variation (13).

The second integral of (13) can be presented as

$$
\begin{gathered}
\int_{R} d^{2} x \partial_{\mu}\left(L \delta x^{\mu}+\frac{\delta L}{\delta\left(\partial_{\mu} u\right)} \delta u\right)= \\
=\int_{R} d^{2} x \partial_{\mu}\left(\frac{\delta L}{\delta\left(\partial_{\mu} u\right)}\left[\delta u+\left(\partial_{\nu} u\right) \delta x^{\nu}\right]-\left[\frac{\delta L}{\delta\left(\partial_{\mu} u\right)}\left(\partial_{\nu} u\right)-\delta_{\nu}^{\mu} L\right] \delta x^{\nu}\right) .
\end{gathered}
$$

Using the total variation (9), Eq. (23), and the energy-momentum tensor

$$
\theta_{\nu}^{\mu}=\frac{\delta L}{\delta\left(\partial_{\mu} u\right)}\left(\partial_{\nu} u\right)-\delta_{\nu}^{\mu} L
$$

the variation (13) yields

$$
\delta S[u, h]=\int_{R} d^{2} x\left(\frac{\delta L}{\delta u}-\partial_{\mu} \frac{\delta L}{\delta\left(\partial_{\mu} u\right)}\right) \delta u+
$$




$$
+\int_{R} d^{2} x \partial_{\mu}\left(\frac{\delta L}{\delta\left(\partial_{\mu} u\right)} \Delta u-\theta_{\nu}^{\mu} \delta x^{\nu}\right) .
$$

Let us consider a continuous (topological) group of coordinate transformation $x \rightarrow x^{\prime}=$ $x^{\prime}(x, a)$, and let the field function $u=u(x)$ admits the representation of this group:

$$
x \rightarrow x^{\prime}, \quad u(x) \rightarrow u^{\prime}\left(x^{\prime}\right) .
$$

The invariance of functional $S[u]$ with respect to (26) means that

$$
\int_{R} d^{2} x^{\prime} L\left[x^{\prime}, u^{\prime}, \partial u^{\prime} / \partial x^{\prime}\right]=\int_{R} d^{2} x L[x, u, \partial u / \partial x] .
$$

The transformation (26) constitutes a group. Therefore, infinitesimal forms of transformations (26) are

$$
\Delta x^{\nu}=X_{s}^{\nu} \delta a^{s}, \quad \Delta u=Y_{s} \delta a^{s}, \quad(\nu=0,1, \quad s=1, . ., m),
$$

where $X_{s}^{\nu}$ and $Y_{s}$ are the generators of the group of transformation correspondingly in the coordinate and the field representations. The index $s=1, . ., m$ is defined by the representation of the group.

For simple examples of transformation of the coordinate, time or scalar field, we have

$$
\begin{gathered}
\Delta x^{\nu}=X^{\nu} \delta a, \quad(\nu=0,1), \\
\Delta u=Y \delta a .
\end{gathered}
$$

Noether's theorem states that every continuous transformation of coordinate (28) and field function (29), which ensures that the variation of the action is zero admits a conservation law in the form of a continuity equation [30, 31]. Substitution of (28), (29), and (15) into (25) gives

$$
\delta S[u, h]=\int_{R} d^{2} x \partial_{\mu}\left(\frac{\delta L}{\delta\left(\partial_{\mu} u\right)} Y-\theta_{\nu}^{\mu} X^{\nu}\right) \delta a .
$$

In view of the fact that the variation of the parameter, $\delta a$, is arbitrary, from (30) we get the conservation law

$$
\partial_{\mu} J^{\mu}=0,
$$

where

$$
J^{\mu}=\frac{\delta L}{\delta\left(\partial_{\mu} u\right)} Y-\theta_{\nu}^{\mu} X^{\nu},
$$


is the Noether's currents and $\theta_{\nu}^{\mu}$ is defined in (24). Equation (31) means that there exists conservation law. Nontriviality of equations (31) and (32) is that $L$ and $\theta_{\nu}^{\mu}$ have nonlocal interaction and memory.

\section{Application of the Noether's theorem for long-range interaction and long-term memory}

\subsection{Lagrangian functional and energy-momentum tensor}

For $\mu=0,1, x^{0}=t$, and $x^{1}=r$, the Euler-Lagrange functional equation (15) is

$$
\frac{\delta L\left[x, u, \partial_{t} u, \partial_{r} u\right]}{\delta u(x)}-\frac{\partial}{\partial t} \frac{\delta L\left[x, u, \partial_{t} u, \partial_{r} u\right]}{\delta\left(\partial_{t} u(x)\right)}-\frac{\partial}{\partial r} \frac{\delta L[x, u, \partial u]}{\delta\left(\partial_{r} u(x)\right)}=0 .
$$

The time and space variables in action can be separated to consider the field with power-law memory and long-range interaction. Let $K_{0}(x, y)$ and $K_{1}(x, y)$ have the form

$$
\begin{aligned}
& K_{0}(x, y)=\delta\left(r-r^{\prime}\right) \mathcal{K}_{0}\left(t, t^{\prime}\right), \\
& K_{1}(x, y)=\delta\left(t-t^{\prime}\right) \mathcal{K}_{1}\left(r, r^{\prime}\right),
\end{aligned}
$$

and

$$
U(u(x), u(y))=U(u(x)) \delta(x-y),
$$

where $x=(t, r)$, and $y=\left(t^{\prime}, r^{\prime}\right)$. Then the Lagrangian functional is

$$
L\left[x, u, \partial_{t} u, \partial_{r} u\right]=\frac{1}{2} \int d t^{\prime} \mathcal{K}_{0}\left(t, t^{\prime}\right) \partial_{t} u \partial_{t^{\prime}} u-\frac{1}{2} \int d r^{\prime} \mathcal{K}_{1}\left(r, r^{\prime}\right) \partial_{r} u \partial_{r^{\prime}} u-U(u) .
$$

To present the long-term memory and long-range interaction, consider the kernels $\mathcal{K}_{0}\left(t, t^{\prime}\right)$ and $\mathcal{K}_{1}\left(r, r^{\prime}\right)$ in the power-law forms

$$
\mathcal{K}_{1}\left(r, r^{\prime}\right)=\mathcal{K}_{1}\left(\left|r-r^{\prime}\right|\right)=\frac{-g}{\cos (\pi \alpha / 2) \Gamma(2-\alpha)} \frac{1}{\left|r-r^{\prime}\right|^{\alpha-1}}, \quad(1<\alpha<2),
$$

and

$$
\mathcal{K}_{0}\left(t, t^{\prime}\right)= \begin{cases}\mathcal{M}\left(t-t^{\prime}\right), & 0<t^{\prime}<t \\ 0, & t^{\prime}>t, \quad t^{\prime}<0\end{cases}
$$


with

$$
\mathcal{M}\left(t-t^{\prime}\right)=\frac{1}{\Gamma(1-\beta)} \frac{1}{\left(t-t^{\prime}\right)^{\beta}}, \quad(0<\beta<1),
$$

Then the variational derivatives of Lagrangian functional are

$$
\begin{gathered}
\frac{\delta L}{\delta u}=-\frac{\partial U(u(t, r))}{\partial u(t, r)}, \\
\frac{\delta L}{\delta\left(\partial_{t} u\right)}={ }_{0}^{C} D_{t}^{\beta} u(t, r), \quad(0<\beta<1), \\
\frac{\delta L}{\delta\left(\partial_{r} u\right)}=-g \frac{\partial^{\alpha-1}}{\partial|r|^{\alpha-1}} u(t, r), \quad(1<\alpha<2),
\end{gathered}
$$

where ${ }_{0}^{C} D_{t}^{\beta}$ is Caputo derivative [14, 12] defined by

$$
{ }_{0}^{C} D_{t}^{\beta} u(t, r)=\frac{1}{\Gamma(n-\beta)} \int_{0}^{t} \frac{d \tau}{(t-\tau)^{\beta-n+1}} \frac{\partial^{n} u(\tau, r)}{\partial \tau^{n}}, \quad(n-1<\beta<n) .
$$

Substitution of (41), (42) and (43) into (33) gives the fractional field Euler-Lagrange equation

$$
{ }_{0}^{C} D_{t}^{\beta+1} u(t, r)-g \frac{\partial^{\alpha}}{\partial|r|^{\alpha}} u(t, r)+\frac{\partial U(u(t, r))}{\partial u(t, r)}=0, \quad(1<\alpha<2,0<\beta<1) .
$$

For $\alpha=2$ and $\beta=1$, Eq. (44) is the usual field equation

$$
\partial_{t}^{2} u(t, r)-g \partial_{r}^{2} u(t, r)+\frac{\partial U(u(t, r))}{\partial u(t, r)}=0
$$

For the potential $U(u(t, r))=-\cos u(t, r)$, equation (45) gives the sine-Gordon equation,

$$
\partial_{t}^{2} u(t, r)-\partial_{r}^{2} u(t, r)+\sin u(t, r)=0,
$$

and Eq. (44) is a spatio-temporal fractional sine-Gordon equation

$$
\partial_{t}^{\beta} u-\partial_{|r|}^{\alpha} u+\sin u=0
$$

where we used for abreviation simplified notation for fractional derivatives. For the case $\beta=2$, the equation was obtained in [20].

The energy-momentum tensor $\theta_{\nu}^{\mu}$, can be presented in the form

$$
\theta_{\nu}^{\mu}=\theta \delta_{\mu \nu}+\tau_{\nu}^{\mu},
$$


where the first term is the diagonal part

$$
\theta=\theta_{0}^{0}+\theta_{1}^{1}=\frac{\delta L}{\delta\left(\partial_{t} u\right)}\left(\partial_{t} u\right)+\frac{\delta L}{\delta\left(\partial_{r} u\right)}\left(\partial_{r} u\right)-2 L=U(u(t, r)) .
$$

This represents a pressure-like quantity. The second term in the rigt hand side of (48) is a nondiagonal of $\theta_{\nu}^{\mu}$ :

$$
\tau_{\nu}^{\mu}=\frac{\delta L}{\delta\left(\partial_{\mu} u\right)}\left(\partial_{\nu} u\right)-\frac{\delta L}{\delta\left(\partial_{\kappa} u\right)}\left(\partial_{\kappa} u\right) \delta_{\nu}^{\mu}
$$

where

$$
\begin{gathered}
\tau_{0}^{0}=\tau_{1}^{1}=0, \\
\tau_{1}^{0}=\frac{\delta L}{\delta\left(\partial_{t} u\right)}\left(\partial_{r} u\right)=\left[{ }_{0}^{C} D_{t}^{\beta} u(t, r)\right] \partial_{r} u(t, r), \\
\tau_{0}^{1}=\frac{\delta L}{\delta\left(\partial_{r} u\right)}\left(\partial_{t} u\right)=-g\left[\frac{\partial^{\alpha-1}}{\partial|r|^{\alpha-1}} u(t, r)\right] \partial_{t} u(t, r) .
\end{gathered}
$$

For $r \in \mathbb{R}^{3}$ the spatial components of $\tau_{\nu}^{\mu}$, represent shear stress tensor. The value $\theta$ represents a pressure-like quantity, normal stress.

\subsection{Homogeneity in time}

The homogeneity in time means invariance of action with respect to the transformation

$$
t \rightarrow t^{\prime}=t+a, \quad r \rightarrow r, \quad u \rightarrow u
$$

Then the infinitesimal transformations are

$$
\Delta x^{\mu}=\delta_{0}^{\mu} \delta a, \quad \Delta u=0, \quad(\mu=0,1)
$$

with the generators

$$
X^{\mu}=\delta_{0}^{\mu}, \quad Y=0, \quad(\mu=0,1)
$$

The Noether's current has two components

$$
J^{\mu}=-\theta_{0}^{\mu}=-\left(\frac{\delta L}{\delta\left(\partial_{\mu} u\right)} \partial_{t} u-\delta_{0}^{\mu} L\right), \quad(\mu=0,1) .
$$

Using $x^{0}=t$, and $x^{1}=r$, we get the continuity equation

$$
\partial_{t} J^{0}+\partial_{r} J^{1}=0
$$


where

$$
\begin{aligned}
& J^{0}=-\left(\frac{\delta L}{\delta\left(\partial_{t} u\right)}\left(\partial_{t} u\right)-L\right)=-\left(\partial_{t} u(t, r){ }_{0}^{C} D_{t}^{\beta} u(t, r)-L\right)= \\
& =-\left(\frac{1}{2} \partial_{t} u(t, r){ }_{0}^{C} D_{t}^{\beta} u(t, r)+\frac{1}{2} g \partial_{r} u(t, r) \frac{\partial^{\alpha-1}}{\partial|r|^{\alpha-1}} u(t, r)+U\right),
\end{aligned}
$$

and

$$
J^{1}=-\frac{\delta L}{\delta\left(\partial_{r} u\right)}\left(\partial_{t} u\right)=g \partial_{t} u(t, r) \frac{\partial^{\alpha-1}}{\partial|r|^{\alpha-1}} u(t, r) .
$$

As a result, the continuity equation is

$$
\partial_{t}\left(\frac{1}{2} \partial_{t} u{ }_{0}^{C} D_{t}^{\beta} u+g \frac{1}{2} \partial_{r} u \frac{\partial^{\alpha-1}}{\partial|r|^{\alpha-1}} u+U\right)-\partial_{r}\left(g \partial_{t} u \frac{\partial^{\alpha-1}}{\partial|r|^{\alpha-1}} u\right)=0
$$

where $u=u(t, r)$. Here

$$
\mathcal{H}=\frac{1}{2} \partial_{t} u{ }_{0}^{C} D_{t}^{\beta} u+\frac{1}{2} g \partial_{r} u \frac{\partial^{\alpha-1}}{\partial|r|^{\alpha-1}} u+U
$$

is a fractional generalization of density of energy (density of Hamiltonian), and

$$
\mathcal{P}=-g \partial_{t} u \frac{\partial^{\alpha-1}}{\partial|r|^{\alpha-1}} u
$$

is a fractional generalization of density of momentum. For the case $\alpha=2$ and $\beta=1$, we obtain the well-known relations [31]:

$$
\mathcal{H}=\frac{1}{2}\left(\partial_{t} u\right)^{2}+\frac{1}{2} g\left(\partial_{r} u\right)^{2}+U, \quad \mathcal{P}=-g \partial_{t} u \partial_{r} u
$$

The continuity equation (61) can be presented in an usual form of the conservation of energy

$$
\partial_{t} \mathcal{H}+\partial_{r} \mathcal{P}=0
$$

with the fractional generalizations of the energy and momentum given by (62) and (63).

\subsection{Homogeneity of space}

The homogeneity of space means invariance of action with respect to transformations

$$
r \rightarrow r^{\prime}=r+a, \quad t \rightarrow t, \quad u \rightarrow u
$$


The corresponding infinitesimal transformations are presented by

$$
\Delta x^{\mu}=\delta_{1}^{\mu} \delta a, \quad \Delta u=0
$$

with the generators

$$
X^{\mu}=\delta_{1}^{\mu}, \quad Y=0
$$

Then the Noether's current has the following two components

$$
J^{\mu}=-\theta_{1}^{\mu}=-\left(\frac{\delta L}{\delta\left(\partial_{\mu} u\right)}\left(\partial_{r} u\right)-\delta_{1}^{\mu} L\right), \quad(\mu=0,1) .
$$

Using $x^{0}=t$, and $x^{1}=r$, we get the continuity equation that corresponds to the homogeneity of one-dimensional space

$$
\partial_{t} J^{0}+\partial_{r} J^{1}=0
$$

where

$$
J^{0}=-\frac{\delta L}{\delta\left(\partial_{t} u\right)}\left(\partial_{r} u\right)=-\partial_{r} u(t, r){ }_{0}^{C} D_{t}^{\beta} u(t, r)
$$

and

$$
\begin{gathered}
J^{1}=-\frac{\delta L}{\delta\left(\partial_{r} u\right)}\left(\partial_{r} u\right)+L=g \partial_{r} u(t, r) \frac{\partial^{\alpha-1}}{\partial|r|^{\alpha-1}} u(t, r)+L= \\
=\frac{1}{2} \partial_{t} u(t, r){ }_{0}^{C} D_{t}^{\beta} u(t, r)+\frac{1}{2} g \partial_{r} u(t, r) \frac{\partial^{\alpha-1}}{\partial|r|^{\alpha-1}} u(t, r)-U .
\end{gathered}
$$

As a result, the continuity equation (70) is

$$
-\partial_{t}\left(\partial_{r} u{ }_{0}^{C} D_{t}^{\beta} u\right)+\partial_{r}\left(\frac{1}{2} \partial_{t} u{ }_{0}^{C} D_{t}^{\beta} u+\frac{1}{2} g \partial_{r} u \frac{\partial^{\alpha-1}}{\partial|r|^{\alpha-1}} u-U\right)=0
$$

that can be interpreted as the momentum conservation law in the case of fractional dynamics.

For $\beta=1$ and $\alpha=2$, equation (73) gives

$$
-\partial_{t}\left(\partial_{r} u \partial_{t} u\right)+\partial_{r}\left(\frac{1}{2}\left(\partial_{t} u\right)^{2}+\frac{1}{2} g\left(\partial_{r} u\right)^{2}-U\right)=0
$$

Note that, in general, for integer and fractional values of $\alpha$ and $\beta, J_{1}^{0} \neq J_{0}^{1}$, and the energymomentum tensor $\theta_{\nu}^{\mu}$ is not a symmetric with respect to $\mu$ and $\nu$. 


\subsection{Field translation invariance}

For the case $U(u)=0$, the action (18) is invariant with respect to transformations

$$
x^{\mu} \rightarrow x^{\prime \mu}=x^{\mu}, \quad u \rightarrow u+a .
$$

The infinitesimal transformations are presented as

$$
\Delta x^{\mu}=0, \quad \Delta u=\delta a
$$

with the generators

$$
X^{\mu}=0, \quad Y=1
$$

The Noether's current

$$
J^{\mu}=\frac{\delta L}{\delta\left(\partial_{\mu} u\right)}, \quad(\mu=1,2)
$$

has components

$$
\begin{gathered}
J^{0}=\frac{\delta L}{\delta\left(\partial_{t} u\right)}={ }_{0}^{C} D_{t}^{\beta} u(t, r), \quad(0<\beta<1), \\
J^{1}=\frac{\delta L}{\delta\left(\partial_{r} u\right)}=-g \frac{\partial^{\alpha-1}}{\partial|r|^{\alpha-1}} u(t, r), \quad(1<\alpha<2) .
\end{gathered}
$$

As a result, the continuity equation

$$
\partial_{\mu} J^{\mu}=\partial_{t} J^{0}+\partial_{r} J^{1}=0
$$

has the form

$$
\partial_{t}{ }_{0}^{C} D_{t}^{\beta} u(t, r)-g \partial_{r} \frac{\partial^{\alpha-1}}{\partial|r|^{\alpha-1}} u(t, r)=0, \quad(1<\alpha<2,0<\beta<1) .
$$

For $\alpha=2$ and $\beta=1$, we get

$$
\partial_{t}^{2} u(t, r)-g \partial_{r}^{2} u(t, r)=0,
$$

which is the usual field equation (45) for $U(u)=0$.

Note that [14]

$$
\partial_{t}{ }_{0}^{C} D_{t}^{\beta} u(t, r)={ }_{0}^{C} D_{t}^{1+\beta} u(t, r)+\frac{t^{-\beta}}{\Gamma(1-\beta)} \partial_{t} u(0, r) .
$$


To have the relation $\partial_{t}{ }_{0}^{C} D_{t}^{\beta} u(t, r)={ }_{0}^{C} D_{t}^{1+\beta} u(t, r)$, the initial conditions $\partial_{t} u(0, r)=0$ should be applied. In general, equation (81) cannot be presented as

$$
{ }_{0}^{C} D_{t}^{1+\beta} u(t, r)-g \frac{\partial^{\alpha}}{\partial|r|^{\alpha}} u(t, r)=0, \quad(1<\alpha<2,0<\beta<1),
$$

which is the fractional field equation (44) for $U(u)=0$.

This shows that the conservation law (81), in general, doesn't coincide with the field equation as it is happened for integer derivatives, unless, we use special boundary and initial conditions.

\section{Chain with long-range interaction}

It was shown in [20, 21, 22, 24, 25] how the long-range interaction between different oscillators can be described by the fractional differential equations in the continuous medium limit. In this section, the Noether's theorem will be applied to such kind of systems.

\subsection{Equation of motion and Noether's currents}

Let us define the action as

$$
S\left[u_{n}\right]=\int_{-\infty}^{+\infty} d t\left(\sum_{n=-\infty}^{+\infty}\left[\frac{1}{2} \dot{u}_{n}^{2}(t)-V\left(u_{n}(t)\right)\right]-\sum_{\substack{n, m=-\infty \\ m \neq n}}^{+\infty} U\left(u_{n}(t), u_{m}(t)\right)\right)
$$

where $u_{n}$ is displacement of the $n$-th oscillator from the equilibrium,

$$
U\left(u_{n}(t)\right)=\frac{1}{4} g_{0} J_{\alpha}(|n-m|)\left(u_{n}(t)-u_{m}(t)\right)^{2}
$$

and

$$
J_{\alpha}(|n-m|)=\frac{1}{|n-m|^{\alpha+1}}, \quad(\alpha>0) .
$$

The Lagrangian of the chain is

$$
\mathcal{L}=\sum_{n=-\infty}^{+\infty}\left[\frac{1}{2} \dot{u}_{n}^{2}(t)-V\left(u_{n}(t)\right)\right]-\frac{1}{4} g_{0} \sum_{\substack{n, m=-\infty \\ m \neq n}}^{+\infty} J_{\alpha}(|n-m|)\left(u_{n}(t)-u_{m}(t)\right)^{2}
$$


The equation of motion

$$
\frac{\partial \mathcal{L}}{\partial u_{n}(t)}-\frac{d}{d t} \frac{\partial \mathcal{L}}{\partial \dot{u}_{n}(t)}=0
$$

for Lagrangian (

$$
\frac{d^{2}}{d t^{2}} u_{n}(t)+\frac{\partial V\left(u_{n}\right)}{\partial u_{n}(t)}+g_{0} \sum_{\substack{n, m=-\infty \\ m \neq n}}^{+\infty} J_{\alpha}(|n-m|)\left[u_{m}(t)-u_{n}(t)\right]=0
$$

A continuous limit of equation (90) can be defined by a transform operation from $u_{n}(t)$ to $u(x, t)[20,21,22,24,25]$. First, define $u_{n}(t)$ as Fourier coefficients of some function $\hat{u}(k, t)$, $k \in[-K / 2, K / 2]$, i.e.

$$
\hat{u}(t, k)=\sum_{n=-\infty}^{+\infty} u_{n}(t) e^{-i k x_{n}}=\mathcal{F}_{\Delta}\left\{u_{n}(t)\right\},
$$

where $x_{n}=n \Delta x$, and $\Delta x=2 \pi / K$ is a distance between nearest particles in the chain, and

$$
u_{n}(t)=\frac{1}{K} \int_{-K / 2}^{+K / 2} d k \hat{u}(t, k) e^{i k x_{n}}=\mathcal{F}_{\Delta}^{-1}\{\hat{u}(t, k)\} .
$$

Secondly, in the limit $\Delta x \rightarrow 0(K \rightarrow \infty)$ replace $u_{n}(t)=(2 \pi / K) u\left(x_{n}, t\right) \rightarrow u(x, t) d x$, and $x_{n}=n \Delta x=2 \pi n / K \rightarrow x$. In this limit, Eqs. (91), (92) are transformed into the integrals

$$
\begin{gathered}
\tilde{u}(t, k)=\int_{-\infty}^{+\infty} d x e^{-i k x} u(t, x)=\mathcal{F}\{u(t, x)\}=\lim _{\Delta x \rightarrow 0} \mathcal{F}_{\Delta}\left\{u_{n}(t)\right\}, \\
u(t, x)=\frac{1}{2 \pi} \int_{-\infty}^{+\infty} d k e^{i k x} \tilde{u}(t, k)=\mathcal{F}^{-1}\{\tilde{u}(t, k)\}=\lim _{\Delta x \rightarrow 0} \mathcal{F}_{\Delta}^{-1}\{\hat{u}(t, k)\} .
\end{gathered}
$$

Applying (91) to (90) and performing the limit (93), we obtain

$$
\frac{\partial^{2} u(t, x)}{\partial t^{2}}+g_{\alpha} \frac{\partial^{\alpha} u(t, x)}{\partial|x|^{\alpha}}+\frac{\partial V(u)}{\partial u(t, r)}=0, \quad(0<\beta<2,1<\alpha<2),
$$

where

$$
g_{\alpha}=2 g_{0}(\Delta x)^{\alpha} \Gamma(-\alpha) \cos \left(\frac{\pi \alpha}{2}\right)
$$

is the renormalized constant. Equations (95) were considered in [20, 21, 22, 24, 25].

Consider a continuous transformation of time and field

$$
t \rightarrow t^{\prime}, \quad u_{n}(t) \rightarrow u_{n}^{\prime}\left(t^{\prime}\right)
$$


which form a continuous group with generators $X$ and $Y_{n}$ such that the infinitesimal transformations are

$$
\Delta t=X \delta a, \quad \Delta u_{n}=Y_{n} \delta a
$$

The corresponding Noether's current is

$$
J=\sum_{n=-\infty}^{+\infty} \frac{\partial \mathcal{L}}{\partial \dot{u}_{n}(t)} Y_{n}-\theta X
$$

where

$$
\theta=\sum_{n=-\infty}^{+\infty} \frac{\partial \mathcal{L}}{\partial \dot{u}_{n}(t)} \dot{u}_{n}(t)-\mathcal{L}
$$

is the energy. Then

$$
J=\sum_{n=-\infty}^{+\infty} \frac{\partial \mathcal{L}}{\partial \dot{u}_{n}(t)}\left[Y_{n}-X \dot{u}_{n}(t)\right]+\mathcal{L} X
$$

The equation of conservation law is

$$
\frac{d}{d t} J=0
$$

In the next subsections, we consider examples of the conservation laws.

\subsection{Homogeneity of time}

The homogeneity of time means the invariance of action with respect to the transformation (compare to (154))

$$
t \rightarrow t+a, \quad u_{n} \rightarrow u_{n}
$$

Its infinitesimal form is

$$
\Delta t=\delta a, \quad \Delta u_{n}=0
$$

with generators

$$
X=1, \quad Y_{n}=0
$$

Then the Noether's current is

$$
J_{t}=-\theta=-\sum_{n=-\infty}^{+\infty} \frac{\partial \mathcal{L}}{\partial \dot{u}_{n}(t)} \dot{u}_{n}(t)+\mathcal{L}=-\sum_{n=-\infty}^{+\infty} \dot{u}_{n}^{2}(t)+\mathcal{L}=
$$




$$
=-\sum_{n=-\infty}^{+\infty} \frac{1}{2} \dot{u}_{n}^{2}(t)-\sum_{n=-\infty}^{+\infty} V\left(u_{n}(t)\right)-\frac{1}{4} g_{0} \sum_{\substack{n, m=-\infty \\ m \neq n}}^{+\infty} J_{\alpha}(|n-m|)\left(u_{n}(t)-u_{m}(t)\right)^{2} \equiv-H,
$$

where $H$ is the Hamiltonian. The conservation law $d H / d t=0$ is in the continuous limit gives the equation

$$
\frac{d}{d t} \int_{-\infty}^{\infty} d r\left[\frac{1}{2}\left[\partial_{t} u(t, r)\right]^{2}+V(u(t, r))+\frac{1}{2} g_{0}, u(t, r) \frac{\partial^{\alpha}}{\partial|r|^{\alpha}} u(t, r)\right]=\frac{d}{d t} \int_{-\infty}^{+\infty} d r \mathcal{H}=0
$$

where $1<\alpha<2$, and the density $\mathcal{H}$ of Hamiltonian (62) is introduced.

One can compare this equation with equation (65) for $\beta=1$. Both results coincide if the boundary conditions

$$
\lim _{r \rightarrow \pm \infty} \mathcal{P}=0
$$

is applied.

\subsection{Translation invariance}

For $V\left(u_{n}\right)=0$, the action (85) is invariant with respect to the transformations

$$
t \rightarrow t, \quad u_{n} \rightarrow u_{n}+a,
$$

or in the infinitesimal form

$$
\Delta t=0, \quad \Delta u_{n}=\delta a,
$$

with the generators

$$
X=0, \quad Y_{n}=1 .
$$

Then the Noether's current is

$$
J_{r}=\sum_{n=-\infty}^{+\infty} \frac{\partial \mathcal{L}}{\partial \dot{u}_{n}(t)}=\sum_{n=-\infty}^{+\infty} \dot{u}_{n}(t) \equiv P
$$

where $P$ is the tolal momentum. The conservation law is $d P / d t=0$.

The continuous limit of this conservation law gives the equation

$$
\frac{d}{d t} \int_{-\infty}^{+\infty} d r \partial_{t} u(t, r)=0
$$


Let us compare this equation with Eq. (81) that is derived for scalar field $u(t, r)$. Integration of (81) with respect to coordinate $r$ gives

$$
\partial_{t} \int_{-\infty}^{+\infty} d r{ }_{0}^{C} D_{t}^{\beta} u(t, r)-g \int_{-\infty}^{+\infty} d r \partial_{r} \frac{\partial^{\alpha-1}}{\partial|r|^{\alpha-1}} u(t, r)=0, \quad(1<\alpha<2,0<\beta<1) .
$$

Then

$$
\partial_{t} \int_{-\infty}^{+\infty} d r{ }_{0}^{C} D_{t}^{\beta} u(t, r)-g\left(\frac{\partial^{\alpha-1}}{\partial|r|^{\alpha-1}} u(t, r)\right)_{-\infty}^{+\infty}=0
$$

As a result, equation (81) coincides with (111), for $\beta=1$, if we use the boundary conditions

$$
\lim _{r \rightarrow \pm \infty} \frac{\partial^{\alpha-1}}{\partial|r|^{\alpha-1}} u(t, r)=0
$$

\section{Fractional Hamilton's equations}

\subsection{De Donder-Weyl Hamiltonian}

The idea of variation of fields based on a manifestly covariant version of the Hamiltonian formalism in field theory known in the calculus of variation of multiple integrals [39, 40] has been proposed by M. Born and H. Weyl [41]. The mathematical study of geometrical structures

underlying the related aspects of the calculus of variations and classical field theory has been undertaken recently by many authors (see for example [40, 42, 43]).

The De Donder-Weyl Hamiltonian form of the field equations [39] are

$$
\partial_{\mu} u(x)=\frac{\partial \mathcal{H}}{\partial \pi^{\mu}}, \quad \partial_{\mu} \pi^{\mu}(x)=-\frac{\partial \mathcal{H}}{\partial u},
$$

where

$$
\pi^{\mu}=\frac{\partial \mathcal{L}}{\partial\left(\partial_{\mu} u\right)}
$$

is called the multi-momenta, and

$$
\mathcal{H}\left(u, \pi^{\mu}\right)=\left(\partial_{\mu} u\right) \pi^{\mu}-\mathcal{L}
$$

is called the De Donder-Weyl Hamiltonian density function, $\mathcal{L}=\mathcal{L}\left(u, \partial_{\mu} u\right)$ is Lagrangian density. These equations are known to be equivalent to the Euler-Lagrange field equations if $\mathcal{L}$ is regular in the sense that

$$
\operatorname{det}\left[\frac{\partial^{2} \mathcal{L}}{\partial\left(\partial_{\mu} u\right) \partial\left(\partial_{\mu} u\right)}\right] \neq 0
$$




\subsection{Hamilton's equations of integer order}

Let us consider Hamiltonian systems in the extended phase space of coordinates $\left(x^{\mu}, u, \pi^{\mu}\right)$. The evolution of fields is defined by stationary states of the action functional

$$
S[u, \pi]=\int\left[\pi^{\mu} \partial_{\mu} u-\mathcal{H}(u, \pi)\right] d^{2} x
$$

where the Hamiltonian density $\mathcal{H}$ is defined by (117), both $u$ and $\pi$ are assumed to be independent functions of $x=(t, r)$. In classical field theory, the evolution of the field $u(x)$ is derived by finding the condition for which the action integral (119) is stationary (a minimum or a saddle point). The action functional (119) can be rewritten as

$$
S[u, \pi]=\int \omega_{\mu}
$$

where

$$
\omega_{\mu}=\pi^{\mu} d u-\mathcal{H}(u, \pi) d x^{\mu}
$$

is the Poincare-Cartan 1-form or the action 1-form.

The stationary action condition $\delta S=0$ leads to

$$
d \omega_{\mu}=0 .
$$

Here the exterior derivative is

$$
d=\left(d x^{\nu}\right) D_{x^{\nu}}+(d u) D_{u}+\left(d \pi^{\nu}\right) D_{\pi^{\nu}},
$$

where we put new notation for the derivatives

$$
D_{x^{\nu}}=\frac{\partial}{\partial x^{\nu}}, \quad D_{u}=\frac{\partial}{\partial u}, \quad D_{\pi^{\nu}}=\frac{\partial}{\partial \pi^{\nu}} .
$$

The De Donder-Weyl Hamiltonian form of field equations in (122) can be obtained from the condition that the derivative is exterior one. This condition is equivalent to the stationary action principle $\delta S[u, \pi]=0$. Then Eq. (121) gives

$$
d \omega_{\mu}=d\left(\pi^{\mu} d u\right)-d\left(\mathcal{H} d x^{\mu}\right)=\left(D_{x^{\nu}} \pi^{\mu}\right) d x^{\nu} \wedge d u+\left(D_{u} \pi^{\mu}\right) d u \wedge d u+\left(D_{\pi^{\nu}} \pi^{\mu}\right) d \pi^{\nu} \wedge d u-
$$




$$
-\left(D_{x^{\nu}} \mathcal{H}\right) d x^{\nu} \wedge d x^{\mu}-\left(D_{u} \mathcal{H}\right) d u \wedge d x^{\mu}-\left(D_{\pi^{\nu}} \mathcal{H}\right) d \pi^{\nu} \wedge d x^{\mu} .
$$

Using $d \pi \wedge d x=-d x \wedge d \pi$, and $\left(D_{x^{\nu}} \mathcal{H}\right), D_{u} \pi=0$, we get

$$
d \omega=\left(D_{x} \pi+D_{u} \mathcal{H}\right) d x \wedge d u-\left(D_{\pi} \pi d u-D_{\pi} \mathcal{H} d x\right) \wedge d \pi,
$$

where the matrix form of the equation is used. The relation $D_{\pi} \pi=1$ gives

$$
d \omega=\left(D_{x} \pi+D_{u} \mathcal{H}\right) d x \wedge d u-\left(d u-D_{\pi} \mathcal{H} d x\right) \wedge d \pi .
$$

From (122), we have

$$
d u-D_{\pi} \mathcal{H} d x=0, \quad D_{x} \pi=-D_{u} \mathcal{H} .
$$

As the result, we obtain

$$
\frac{\partial u}{\partial x^{\mu}}=D_{\pi^{\mu}} \mathcal{H}, \quad \frac{\partial \pi^{\mu}}{\partial x^{\mu}}=-D_{u} \mathcal{H},
$$

which are the well-known De Donder-Weyl Hamilton's equations (115).

\subsection{Fractional Hamilton's equations}

The fractional generalization of the form (121) can be defined [37, 35] by

$$
\omega(\alpha)=\pi d_{s}^{\alpha} u-\mathcal{H}(u, \pi) d_{s}^{\alpha} x .
$$

It will be called the fractional Poincare-Cartan 1-form or simply the fractional action 1-form. We can consider the fractional exterior derivative of the form (130), and use

$$
d^{\alpha} \omega(\alpha)=0
$$

to obtain the fractional field equations. Here the fractional exterior derivative is

$$
d^{\alpha}=d_{s}^{\alpha} x^{\nu} D_{x^{\nu}}^{\alpha}+d_{s}^{\alpha} u D_{u}^{\alpha}+d_{s}^{\alpha} \pi^{\nu} D_{\pi^{\nu}}^{\alpha}, \quad d_{s}^{\alpha} x=\Gamma^{-1}(\alpha+1) d^{\alpha} x^{\alpha},
$$

where $D_{x^{\nu}}^{\alpha}, D_{u}^{\alpha}, D_{\pi^{\nu}}^{\alpha}$ can be fractional derivatives of different types [14]. For example, for $x^{\mu} \in \mathbb{R}^{2}$, such that $x=(t, r)$, we use

$$
D_{x}^{\alpha}=\left({ }_{t_{0}}^{C} D_{t}^{\alpha_{0}}, D_{r}^{\alpha_{1}}\right)
$$


where ${ }_{t_{0}}^{C} D_{t}^{\alpha_{0}}$ is Caputo fractional derivative, and $D_{r}^{\alpha_{1}}$ is the Riesz derivative. Fractional differential forms have been suggested in [37] and it is used to describe dynamical systems [35, 38].

Then, by some transformations (see Appendix), one can obtain

$$
d^{\alpha} \omega(\alpha)=\left[D_{x}^{\alpha} \pi+D_{u}^{\alpha} \mathcal{H}\right] d_{s}^{\alpha} x \wedge d_{s}^{\alpha} u-\left[\frac{\pi^{1-\alpha}}{\Gamma(2-\alpha)} d_{s}^{\alpha} u-D_{\pi}^{\alpha} \mathcal{H} d_{s}^{\alpha} x\right] \wedge d_{s}^{\alpha} \pi
$$

Using (133) and (131), we get

$$
\frac{\pi^{1-\alpha}}{\Gamma(2-\alpha)} d_{s}^{\alpha} u-D_{\pi}^{\alpha} \mathcal{H} d_{s}^{\alpha} x=0, \quad D_{x}^{\alpha} \pi=-D_{u}^{\alpha} \mathcal{H}
$$

For the case $u=u(x)$,

$$
d_{s}^{\alpha} u=d_{s}^{\alpha} x D_{x}^{\alpha} u
$$

As the result, we obtain

$$
D_{x^{\mu}}^{\alpha} u=\left(\pi^{\mu}\right)^{\alpha-1} \Gamma(2-\alpha) D_{\pi^{\mu}}^{\alpha} \mathcal{H}, \quad D_{x^{\mu}}^{\alpha} \pi^{\mu}=-D_{u}^{\alpha} \mathcal{H}
$$

These equations are the fractional generalization of De Donder-Weyl Hamilton's equations (129).

As an example, we can consider

$$
\mathcal{H}(u, \pi)=\frac{2-\alpha}{2}\left(\pi^{0}\right)^{2}-\frac{2-\alpha}{2}\left(\pi^{1}\right)^{2}+U(u)
$$

Using

$$
D_{\pi^{\nu}}^{\alpha}\left(\pi^{\mu}\right)^{\beta}=\frac{\Gamma(\beta+1)}{\Gamma(\beta+1-\alpha)}\left(\pi^{\mu}\right)^{\beta-\alpha} \delta_{\nu}^{\mu},
$$

and $\Gamma(z+1)=z \Gamma(z)$, equations (136) give

$$
\begin{gathered}
D_{t}^{\alpha_{0}} u=\pi^{0}, \quad D_{r}^{\alpha_{1}} u=-\pi^{1}, \\
D_{t}^{\alpha_{0}} \pi_{0}+D_{t}^{\alpha_{1}} \pi_{1}=-D_{u}^{\alpha_{u}} U(u) .
\end{gathered}
$$

After substitution of (139) into (140), we obtain

$$
\left(D_{t}^{\alpha_{0}}\right)^{2} u(t, r)-\left(D_{r}^{\alpha_{1}}\right)^{2} u(t, r)+D_{u}^{\alpha_{u}} U(u)=0 .
$$


For $\alpha_{0}=\alpha_{1}=\alpha_{u}=1$, Eq. (141) is the usual wave equation

$$
\partial_{t}^{2} u(t, r)-\partial_{r}^{2} u(t, r)+\frac{\partial U(u)}{\partial u(t, r)}=0 .
$$

For $U(u)=0$, Eq. (141) has the form

$$
\left(D_{t}^{\alpha_{0}}\right)^{2} u(t, r)-\left(D_{r}^{\alpha_{1}}\right)^{2} u(t, r)=0 .
$$

The solution $u(t, r)$ of this equation is a linear combination of the solutions $u_{1}(t, r)$ and $u_{2}(t, r)$ of the equations

$$
D_{t}^{\alpha_{0}} u_{1}(t, r)-D_{r}^{\alpha_{1}} u_{1}(t, r)=0, \quad D_{t}^{\alpha_{0}} u_{2}(t, r)+D_{r}^{\alpha_{1}} u_{2}(t, r)=0 .
$$

For $\alpha_{1}=1$, there exists a relation between the Dirac solutions and the fractional extension of D'Alembert expression that is considered in [44].

Using the property

$$
D_{x}^{\alpha} D_{x}^{\alpha} u=D_{x}^{2 \alpha} u
$$

and we get the fractional equation

$$
D_{t}^{2 \alpha_{0}} u-D_{r}^{2 \alpha_{1}} u+D_{u}^{\alpha_{u}} U(u)=0 .
$$

For a special case,

$$
U(u)=m^{2} \frac{\Gamma(2)}{\Gamma\left(2+\alpha_{u}\right)} u^{1+\alpha_{u}},
$$

equation (145) gives

$$
D_{t}^{2 \alpha_{0}} u(t, r)-D_{r}^{2 \alpha_{1}} u(t, r)+m^{2} u(t, r)=0 .
$$

This is a fractional generalization of Klein-Gordon equation (see also [20, 33]). Note that Eq. (147) is not Lorentz invariant equations. To obtain fractional relativistic equations, the fractional power of D'Alembertian should be used [45] (see also Sec. 28 of [11]). The causality principle [31, 46] also should be taken into account.

For

$$
U(u)=\sin (u(t, r))
$$


equation (145) gives

$$
D_{t}^{2 \alpha_{0}} u-D_{r}^{2 \alpha_{1}} u+\sin \left(u+\alpha_{u} \pi / 2\right)=0 .
$$

This equation is fractional sine-Gordon equation that was considered in [20] for $\alpha_{0}=1$ and $\alpha_{u}=2$.

\section{Conclusion}

Exploiting a variational principle to obtain fractional dynamics seems to be a fairly powerful tool that permits a universal consideration of situations with fractal time and space. In this paper, we have demonstrate it by deriving different equations of using generalized Noether's theorem. Such equations are similar to the regular conservation laws, although the presence of fractional time derivatives reflects a dissipation. Similar variation of action can be used to derive Hamiltonian type equations although the situation here is not uniquely defined and some freedom of choosing the differential action 1-form leaves different possibilities.

\section{Acknowledge}

This work was supported by the Office of Naval Research, Grant No. N00014-02-1-0056, and the NSF Grant No. DMS-0417800.

\section{References}

[1] E.W. Montroll, M.F. Shlesinger, "The wonderful world of random walks" In: Studies in Statistical Mechanics, Vol. 11. J. Lebowitz, E. Montroll (Eds.), (North-Holland, Amsterdam, 1984) pp.1-121.

[2] J.P. Bouchaud, A. Georges, "Anomalous diffusion in disordered media-statistical mechanisms, models and physical applications" Phys. Rep. 195 (1990) 127-293. 
[3] R.R. Nigmatullin, "The generalized fractals and statistical properties of the pore-space of the sedimentary-rocks" Phys. Stat. Sol. B 153 (1989) 49-57; "Fractional integral and its physical interpretation" Theor. Math. Phys. 90 (1992) 242-251.

[4] R. Gorenflo, F. Mainardi, "Fractional calculus: integral and differential equations of fractional order", in Fractals and Fractional Calculus in Continuum Mechanics (Springer, Wien, 1997), pp.223-276.

[5] V.V. Afanasiev, R.Z. Sagdeev, G.M. Zaslavsky, "Chaotic jets with multifractal space-time random walk " Chaos 1 (1991) 143-159.

[6] R. Metzler, J. Klafter, "The random walk's guide to anomalous diffusion: a fractional dynamics approach" Phys. Rep. 339 (2000) 1-77.

[7] E. Barkai, R. Metzler, J. Klafter, "From continuous time random walks to the fractional Fokker-Planck equation", Phys. Rev. E 61 (2000) 132-138.

[8] G.M. Zaslavsky, "Chaos, fractional kinetics, and anomalous transport" Phys. Rep. 371 (2002) 461-580.

[9] R. Hilfer (Ed.), Applications of Fractional Calculus in Physics, (World Scientific, Singapore, 2000).

[10] B. West, M. Bologna, P. Grigolini, Physics of Fractal Operators (Springer, New York, $2003)$.

[11] S.G. Samko, A.A. Kilbas, O.I. Marichev, Fractional Integrals and Derivatives Theory and Applications (Gordon and Breach, New York, 1993).

[12] I. Podlubny, Fractional Differential Equations (Academic Press, San Diego, 1999).

[13] K. Miller, B. Ross, An Introduction to the Fractional Calculus and Fractional Differential Equations (Wiley, New York, 1993).

[14] A.A. Kilbas, H.M. Srivastava, J.J. Trujillo, Theory and Application of Fractional Differential Equations (Elsevier, Amsterdam, 2006).

[15] M.M. Meerschaert, D.A. Benson, B. Baeumer, "Operator Levy Motion and multiscaling anomalous diffusion", Phys. Rev. E 63 (2001) 1112-1117.

[16] G.M. Zaslavsky, "Multifractional kinetics" Physica A 288 (2000) 431-443. 
[17] M.M. Meerschaert, J. Mortensen, S.W. Wheatcraft, "Fractional vector calculus for fractional advectiondispersion" Physica A 367 (2006) 181-190.

[18] O.P. Agrawal "Formulation of Euler-Lagrange equations for fractional variational problems" J. Math. Anal. Appl. 272 (2002) 368-379;

D. Baleanu, O.P. Agrawal, "Fractional Hamilton formalism within Caputo's derivative" Czechoslovak J. Phys. 56 (2006) 1087-1092.

[19] R. Schumer, D.A. Benson, M.M. Meerschaert, S.W. Wheatcraft, "Eulerian derivation of the fractional advection-dispersion equation" J. Contaminant Hydrol. 48 (2001) 6988.

[20] N. Laskin, G.M. Zaslavsky, "Nonlinear fractional dynamics on a lattice with long-range interactions" Physica A 368 (2006) 38-54 (nlin.SI/0512010).

[21] V.E. Tarasov, G.M. Zaslavsky, "Fractional dynamics of coupled oscillators with long-range interaction" Chaos 16 (2006) 023110; "Fractional dynamics of systems with long-range interaction" Commun. Nonlin. Sci. Numer. Simul. 11 (2006) 885-898 (nlin.PS/0512013).

[22] N. Korabel, G.M. Zaslavsky, V.E. Tarasov, "Coupled oscillators with power-law interaction and their fractional dynamics analogues" Commun. Nonlin. Sci. Numer. Simul. accepted (math-ph/0603074).

[23] V.E. Tarasov, G.M. Zaslavsky "Fractional dynamics of systems with long-range space interaction and temporal memory" Physica A (2007). Preprint math-ph/0702065.

[24] N. Korabel, G.M. Zaslavsky, "Transition to chaos in discrete nonlinear Schrodinger equation with long-range interaction" Preprint math-ph/0607030.

[25] V.E. Tarasov, "Continuous limit of discrete systems with long-range interaction" J. Phys. A 39 (2006) 14895-14910.

[26] J. Cresson, "Fractional embedding of differential operators and Lagrangian systems" Preprint math.DS/0605752.

[27] G.S.F. Frederico, D.F.M. Torres, " A formulation of Noethers theorem for fractional problems of the calculus of variations" Preprint math.OC/0701187.

[28] J. Frohlich, R. Israel, E.H. Lieb, B. Simon, "Phase transitions and reflection positivity. I. General theory and long range lattice models " Commun Math Phys 62 (1978) 1-34; 
H. Brezis, E.H. Lieb, "Long-range atomic potentials in Thomas-Fermi theory" Commun. Math. Phys. 65 (1979) 231-246.

[29] J. Barre, F. Bouchet, T. Dauxois, S. Ruffo, "Large deviation techniques applied to systems with long-range interactions " J. Stat. Phys. 119 (2005) 677-713;

J. Barre, D. Mukamel, S. Ruffo, "Inequivalence of ensembles in a system with long-range interactions" Phys. Rev. Lett. 87 (2001) 030601.

[30] E. Noether, "Invariant variation problems" physics/0503066 M. A. Tavels English translation of "Invariante Variationsprobleme", Nachr. d. Konig. Gesellsch. d. Wiss. zu Gottingen, Math-phys. Klasse, (1918) 235-257; Transport Theory and Statistical Physics 1 (1971) 183-207.

[31] N.N. Bogoliubov, D.V. Shirkov, Introduction to the Theory of Quantized Fields (Wiley, New York, 1980).

[32] F. Riewe, "Nonconservative Lagrangian and Hamiltonian mechanics" Phys. Rev. E 53 (1996) 1890-1899; "Mechanics with fractional derivatives" Phys. Rev. E 55 (1997) 35813592.

[33] D. Baleanu, S.I. Muslih, "Lagrangian formulation of classical fields within RiemannLiouville fractional derivatives" Phys. Scripta 72 (2006) 119-121 (hep-th/0510071);

S.I. Muslih D. Baleanu, E. Rabei, "Hamiltonian formulation of classical fields within Riemann-Liouville fractional derivatives" Phys. Scripta 73 (2006) 436-438;

E.M. Rabei, K.I. Nawafleh, R.S. Hijjawi, S.I. Muslih, D. Baleanu, "The Hamilton formalism with fractional derivatives" J. Math. Anal. Appl. 327 (2007) 891-897.

[34] A.A. Stanislavsky, "Hamiltonian formalism of fractional systems" European Phys. J. B 49 (2006) 93-101.

[35] V.E. Tarasov "Fractional variations for dynamical systems: Hamilton and Lagrange approaches" J. Phys. A 39 (2006) 8409-8425 (math-ph/0606048).

[36] V.E. Tarasov, "Continuous medium model for fractal media" Phys. Lett. A 336 (2005) 167-174 (cond-mat/0506137); "Fractional hydrodynamic equations for fractal media" Ann. Phys. 318 (2005) 286-307 (physics/0602096). 
[37] K. Cottrill-Shepherd, M. Naber, "Fractional differential forms" J. Math. Phys. 42 (2001) 2203-2212 (math-ph/0301013); "Fractional differential forms II" Preprint math-ph/0301016.

[38] V.E. Tarasov, "Fractional generalization of gradient and Hamiltonian systems" J. Phys. A 38 (2005) 5929-5943; "Fractional generalization of gradient systems" Lett. Math. Phys. 73 (2005) 49-58; "Fractional statistical mechanics" Chaos 16 (2006) 033108.

[39] Th. De Donder, Theorie invariantive du calcul des variations (Nuov. ed. Gauthier-Villars, Paris, 1935);

H. Weyl, "Geodesic fields in the calculus of variations" Ann. Math. 36 (1935) 607-29.

[40] H.A. Kastrup, "Canonical theories of Lagrangian dynamical systems in physics" Phys. Rep. 101 (1983) 1-167 and references therein.

[41] M. Born, "On the quantum theory of the electromagnetic field" Proc. Roy. Soc. (London) A 143 (1934) 410-437;

H. Weyl, "Observations on Hilbert's independence theorem and Born's quantization of field equations" Phys. Rev. 46 (1934) 505-508.

[42] G. Giachetta, L. Mangiarotti, G. Sardanashvily, New Lagrangian and Hamiltonian Methods in Field Theory (World Scientific, Singapore 1997).

[43] I.V. Kanatchikov, "Canonical structure of classical field theory in the polymomentum phase space" Rep. Math. Phys. 41 (1998) 49-90 (hep-th/9709229).

[44] T. Pierantozzi, L. Vazquez, "An interpolation between the wave and diffusion equations through the fractional evolution equations Dirac like" J. Math. Phys. 46 (2005) 113512.

[45] C.G. Bollini, J.J. Giambiagi, "Arbitrary powers of DAlembertians and the Huygens principle" J. Math. Phys. 34 (1993) 610-621.

[46] S. Kempfle, "Causality criteria for solutions of linear fractional differential equations" Fractional Calculus and Applied Analysis 1(4) (1998) 351-364;

S. Kempfle, I. Schafer, H. Beyer, "Fractional calculus via functional calculus: Theory and applications" Nonlinear Dynamics 29 (2002) 99-127. 


\section{Appendix}

To prove the proposition (133), we use the rule

$$
D_{x}^{\alpha}(f g)=\sum_{s=0}^{\infty}\left(\begin{array}{l}
\alpha \\
s
\end{array}\right)\left(D_{x}^{\alpha-s} f\right) \frac{\partial^{s} g}{\partial x^{s}},
$$

and the relation 14

$$
\frac{\partial^{s}}{\partial x^{s}}\left[d_{s}^{\alpha} x\right]=0 \quad(s \geq 1)
$$

for integer $s$, where

$$
\left(\begin{array}{l}
\alpha \\
k
\end{array}\right)=\frac{(-1)^{k-1} \alpha \Gamma(k-\alpha)}{\Gamma(1-\alpha) \Gamma(k+1)} .
$$

For example, we have

$$
\begin{aligned}
& d^{\alpha}\left[A_{\mu} d_{s}^{\alpha} x^{\mu}\right]=\sum_{s=0}^{\infty} d_{s}^{\alpha} x^{\nu} \wedge\left(\begin{array}{c}
\alpha \\
s
\end{array}\right)\left(D_{x^{\nu}}^{\alpha-s} A_{\mu}\right) \frac{\partial^{s}}{\partial\left(x^{\nu}\right)^{s}} d_{s}^{\alpha} x^{\mu}= \\
& =d_{s}^{\alpha} x^{\nu} \wedge d_{s}^{\alpha} x^{\mu}\left(\begin{array}{c}
\alpha \\
0
\end{array}\right)\left(D_{x^{\nu}}^{\alpha} A_{\mu}\right)=\left(D_{x^{\nu}}^{\alpha} A_{\mu}\right) d_{s}^{\alpha} x^{\nu} \wedge d_{s}^{\alpha} x^{\mu}
\end{aligned}
$$

As a result,

$$
\begin{gathered}
d^{\alpha} \omega(\alpha)=d^{\alpha}\left(\pi d_{s}^{\alpha} u\right)-d^{\alpha}\left(\mathcal{H} d_{s}^{\alpha} x\right)= \\
=\left(D_{x}^{\alpha} \pi\right) d_{s}^{\alpha} x \wedge d_{s}^{\alpha} u+\left(D_{u}^{\alpha} \pi\right) d_{s}^{\alpha} u \wedge d_{s}^{\alpha} u+\left(D_{\pi}^{\alpha} \pi\right) d_{s}^{\alpha} \pi \wedge d_{s}^{\alpha} u- \\
-\left(D_{x}^{\alpha} \mathcal{H}\right) d_{s}^{\alpha} x \wedge d_{s}^{\alpha} x-\left(D_{u}^{\alpha} \mathcal{H}\right) d_{s}^{\alpha} u \wedge d_{s}^{\alpha} x-\left(D_{\pi}^{\alpha} \mathcal{H}\right) d_{s}^{\alpha} \pi \wedge d_{s}^{\alpha} x .
\end{gathered}
$$

Here $D_{x^{0}}^{\alpha}={ }_{t_{0}}^{C} D_{t}^{\beta}$ is Caputo fractional derivative, and $D_{x^{1}}^{\alpha}=\partial^{\alpha} / \partial|r|^{\alpha}$ is the Riesz fractional derivative [14]. Using

$$
d_{s}^{\alpha} \pi \wedge d_{s}^{\alpha} x=-d_{s}^{\alpha} x \wedge d_{s}^{\alpha} \pi
$$

and $D_{x} \mathcal{H}(u, \pi)=0, D_{u}^{\alpha} \pi=0$ for Riesz and Caputo derivatives, we can rewrite equation (150) in the form

$$
d^{\alpha} \omega(\alpha)=\left[D_{x}^{\alpha} \pi+D_{u}^{\alpha} \mathcal{H}\right] d_{s}^{\alpha} x \wedge d_{s}^{\alpha} u-\left[\left(D_{\pi}^{\alpha} \pi\right) d_{s}^{\alpha} u-\left(D_{\pi}^{\alpha} \mathcal{H}\right) d_{s}^{\alpha} x\right] \wedge d_{s}^{\alpha} \pi
$$

Substitution of

$$
D_{\pi}^{\alpha} \pi=\frac{\pi^{1-\alpha}}{\Gamma(2-\alpha)}
$$

into equation (151) gives (133). 